\title{
High expression of UBE2C is associated with the aggressive progression and poor outcome of malignant glioma
}

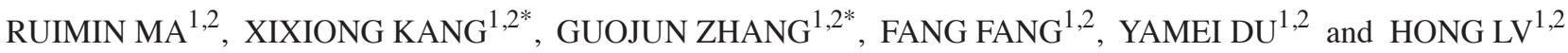 \\ ${ }^{1}$ Laboratory Diagnosis Center, Beijing Tiantan Hospital, Capital Medical University; \\ ${ }^{2}$ Beijing Engineering Research Center of Immunological Reagents Clinical Research, Beijing 100050, P.R. China
}

Received February 5, 2015; Accepted December 18, 2015

DOI: $10.3892 /$ ol.2016.4171

\begin{abstract}
Ubiquitin-conjugating enzyme E2C (UBE2C) is a key regulator of cell cycle progression and is involved in the tumorigenesis of a variety of cancers. Previous studies have demonstrated that $\mathrm{UBE} 2 \mathrm{C}$ is an important factor in the malignant progression of astrocytic tumors. However, the association between UBE2C expression and clinical prognosis of glioma patients has not been defined. In the present study, the expression of UBE2C in gliomas and non-cancerous brain tissues were detected by microarray and immunohistochemical analysis. The association between UBE2C expression and clinicopathological characteristics of the glioma patients was evaluated. The Kaplan-Meier method and multivariate Cox's proportional hazards model were used to analyze the survival time of the patients. The results demonstrated that the expression levels of UBE2C in anaplastic gliomas and glioblastoma (GBM) patients were significantly higher compared to low-grade gliomas, in microarray and immunohistochemistry analysis. A higher UBE2C expression was associated with a significantly decreased overall survival time in patients possessing anaplastic gliomas $(\mathrm{P}<0.01)$ and $G B M s$ $(\mathrm{P}<0.05)$. Multivariate analysis of $80 \mathrm{GBM}$ patients revealed that UBE2C expression was an independent prognostic factor. To the best of our knowledge, the present data suggest for the first time that UBE2C overexpression is strongly associated with an aggressive progression and poor outcome of malignant
\end{abstract}

Correspondence to: Dr Xixiong Kang or Dr Guojun Zhang, Laboratory Diagnosis Center, Beijing Tiantan Hospital, Capital Medical University, 6 Tiantan Xili, Beijing 100050, P.R. China

E-mail: clinical_lab@126.com

E-mail: teacherzhang1@126.com

* Contributed equally

Abbreviations: UBE2C, ubiquitin-conjugating enzyme E2C; GBM, glioblastoma; IDH1, isocitrate dehydrogenase-1; TMZ, temozolomide

Key words: anaplastic glioma, ubiquitin-conjugating enzyme E2C, outcome, biomarker, glioblastoma glioma. Therefore, UBE2C overexpression may be used as a predictor of poor prognosis in patients with malignant glioma.

\section{Introduction}

The most common primary tumor of the central nervous system in adults is glioma, which demonstrates a variety of histological tumor types and World Health Organization (WHO) malignancy grades $(1,2)$. Usually, the treatment strategy for glioma consists of surgery combined with radiation therapy and chemotherapy; however, the prognosis for patients with malignant glioma remains poor (3). In general, a therapeutic strategy is decided according to histopathological results of the tumors (4). The initiation and progression of glioma is generally hypothesized to involve the accumulation of multiple genetic alterations; therefore, the development of novel molecular markers is required to improve glioma classification, the prediction of the prognosis of patients and targeted therapeutic treatment strategies (5).

Ubiquitin-conjugating (E2) enzymes are members of a family of structurally-associated proteins that mediate ubiquitin-dependent proteolysis, including diverse cellular processes, such as cell cycle progression, signal transduction and differentiation $(6,7)$. Ubiquitin-conjugating enzyme E2C (UBE2C) belongs to the E2 family, and is involved in mitotic cyclin destruction and cell cycle progression (8). UBE2C has been implicated in the carcinogenesis of certain tumors $(9,10)$, and is overexpressed in various human cancers, including breast, esophageal, colon, lung, liver and ovarian cancers (11-16).

In previous studies, the upregulation of UBE2C in brain tumors has been reported $(17,18)$. In addition, the expression levels of UBE2C were elevated in high-grade astrocytomas, but not low-grade astrocytomas (18). However, to the best of our knowledge, the prognostic significance of the expression of UBE2C in glioma has not been elucidated. The present study used a large cohort of glioma patients and aimed to perform an analysis of UBE2C expression in glioma tumors and to investigate the association of glioma with clinicopathological variables and the prognosis of patients.

\section{Materials and methods}

Patients and tissue samples. The present study was approved by the Ethics Committee of Beijing Tiantan Hospital (Beijing, 


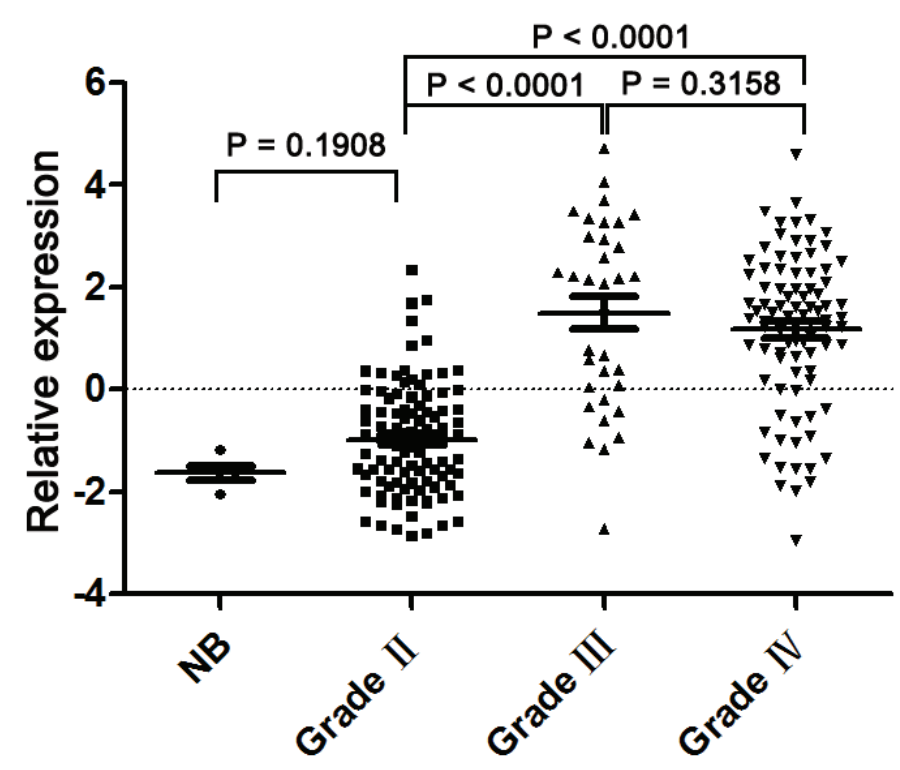

Figure 1. The expression levels of ubiquitin-conjugating enzyme E2C in grade II-IV glioma and non-cancerous brain tissues. NB, non-cancerous brain tissue.

China). Written informed consent was obtained from all patients. Tissue samples were obtained from patients during surgery at Beijing Tiantan Hospital between January 2006 and December 2009. In total, 220 adult patients diagnosed with glioma were enrolled in the present study. The glioma tumor grade of patients was diagnosed histologically according to the 2007 WHO classification (2), as follows: 97 patients possessed grade II disease; 34 patients possessed grade III disease; and 89 patients possessed grade IV disease. Control non-cancerous brain tissue specimens were obtained from 5 patients with craniocerebral injuries.

All patients had undergone prior surgical resection, followed by adjuvant radiation and alkylating agent-based chemotherapy. Post-operative follow-up data was gathered for all patients, and the clinical characteristics and pathological findings, including the age at diagnosis, gender, extent of resection, isocitrate dehydrogenase-1 (IDH1) mutation status and temozolomide (TMZ) chemotherapy, were obtained.

RNA extraction and gene expression profiling. Total RNA was extracted from frozen tumor tissues using the Ambion mirVana $^{\mathrm{TM}}$ miRNA Isolation kit (Thermo Fisher Scientific, Inc., Waltham, MA, USA), according to the manufacturer's protocol. RNA concentration and quality were measured using the NanoDrop ND-1000 spectrophotometer (Thermo Fisher Scientific, Inc., Wilmington, DE, USA), and its integrity was determined using the Agilent Bioanalyzer 2100 (Agilent Technologies, Inc., Santa Clara, CA, USA). Gene expression analysis was performed using Agilent Gene Expression oligo microarrays (Agilent Technologies, Inc.) containing $>41,000$ probe sets for $\sim 27,958$ human genes using the Agilent One-Color Microarray-Based Gene Expression Analysis (catalog no., G4140-90040; Agilent Technologies, Inc.), according to the manufacturer's protocol. Average values of the replicate spots for each gene were background subtracted, normalized, $\log _{2}$-transformed and subjected to additional analysis.
Immunohistochemistry analysis. Formalin-fixed, paraffin-embedded (FFPE), $5 \mu \mathrm{m}$-thick tissue sections were used for immunohistochemical analysis, which was performed using a streptavidin-biotin immunoperoxidase assay, as previously described (19). Immunohistochemical staining was performed using anti-human mouse monoclonal anti-UBE2C antibody (catalog no., ab201979; dilution, 1:100; Abcam, Cambridge, MA, USA). Staining for UBE2C in the nucleus or the cytoplasm was scored as absent, weak, moderate or strong. Absent or weak staining was categorized as negative, while moderate or strong staining was categorized as positive. Controls consisted of tissues without primary antibody and non-cancerous control tissues, and were included in all experiments to ensure the quality of staining. In total, 2 pathologists performed immunohistochemistry scoring independently and blindly.

Statistical analysis. Statistical analyses were performed using a $\chi^{2}$ test or Fisher's exact test with $2 \times 2$ tables. Kaplan-Meier survival time analysis was used to estimate the survival time distributions, and the log-rank test was used to assess the statistical significance between different groups. Multivariate analysis was performed using Cox's proportional hazards model, and the risk ratio and $95 \%$ confidence intervals were calculated for each characteristic. Statistical analysis was performed using SPSS version 13.0 for Windows (SPSS, Inc., Chicago, IL, USA). Two-tailed tests were performed. $\mathrm{P}<0.05$ was considered to indicate a statistically significant difference.

\section{Results}

UBE2C expression in glioma. A microarray was performed to detect the UBE2C mRNA expression in 220 glioma tissues and 5 non-cancerous brain specimens. The present study observed significant differences in the UBE2C expression between non-cancerous brain, low-grade glioma and high-grade glioma tissues. Fig. 1 reveals that the expression levels of UBE2C mRNA in grade II tumor tissues were significantly decreased 

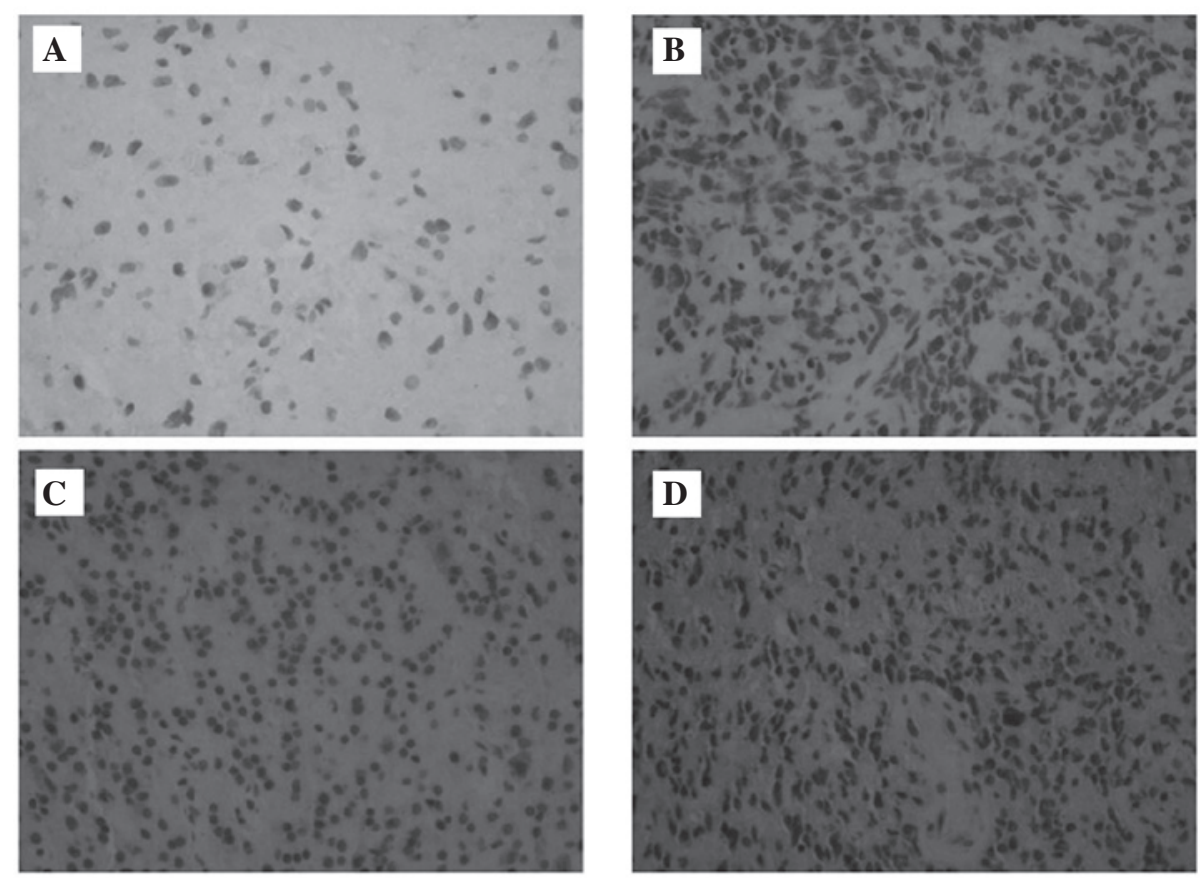

Figure 2. Immunohistochemical expression of UBE2C in glioma tissues of various grades. (A) No expression of UBE2C was observed in non-cancerous brain tissue. (B) A few UBE2C-positive tumor cells were observed in low-grade tumor tissues. A increased staining intensity was detected in (C) anaplastic glioma and (D) glioblastoma tissues. UBE2C, ubiquitin-conjugating enzyme E2C.
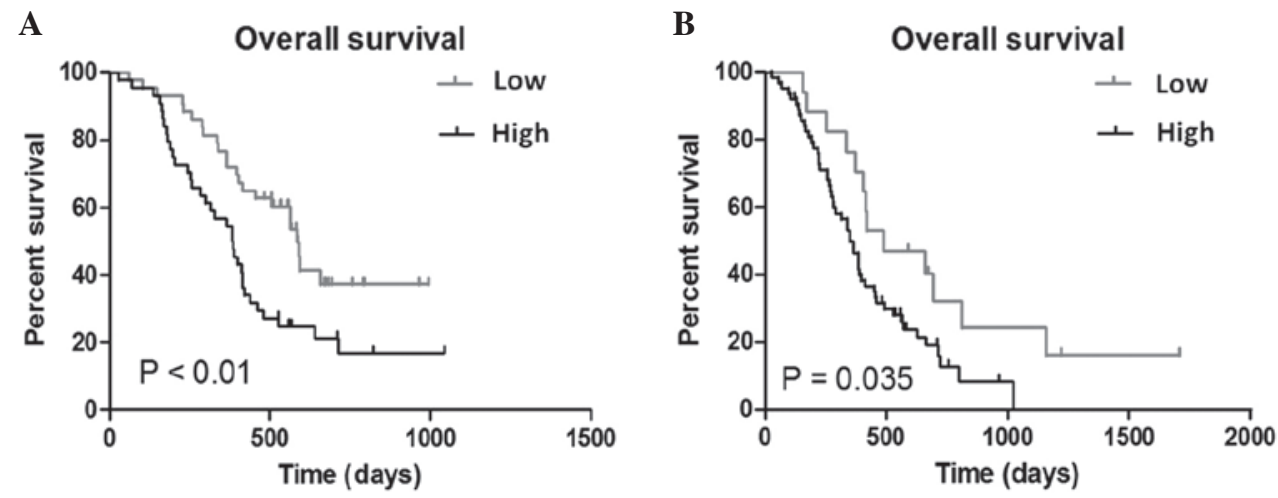

Figure 3. Association between ubiquitin-conjugating enzyme E2C expression and survival time in (A) anaplastic glioma and (B) glioblastoma tissues.

compared to grade III $(\mathrm{P}<0.0001)$ and IV $(\mathrm{P}<0.0001)$ glioma tissues. However, there is no significant difference in UBE2C expression levels between grade III and IV gliomas.

To additionally detect the expression of the UBE2C protein in glioma tissues, immunohistochemistry was performed. As illustrated in Fig. 2, UBE2C was not expressed in non-cancerous brain tissues. There was significant UBE2C protein expression in 46 of 97 (47.4\%) low-grade gliomas. In total, $73.5 \%$ of patients ( 25 out of 34 patients) with anaplastic (grade III) gliomas demonstrated significant UBE2C protein expression, whereas 78.8\% (63 of 80) of glioblastomas (GBM; grade IV) exhibited significant UBE2C staining (2).

Association between UBE2C expression and the clinicopathological parameters. The present study evaluated the association of UBE2C expression with clinicopathological variables (Table I). According to the strength of staining for UBE2C in cells, which was classified as absent, weak, moderate or strong staining, the glioma tissues were divided into two groups, negative and positive. UBE2C expression was not associated with the patient age, patient gender, extent of resection, IDH1 mutation status and TMZ chemotherapy.

Association between UBE2C expression and survival time. The Kaplan-Meier method was performed to elucidate the association of UBE2C expression with the prognosis of glioma patients. The median expression of UBE2C in anaplastic glioma patients was 2.1061 and the median expression of UBE2C in GBM patients was 1.3937. When the median expression values of UBE2C (the relative expression level detected by microarray) was used as the cut-off point, patients with a higher UBE2C expression possessed a significantly decreased overall survival time in patients with anaplastic glioma $(\mathrm{P}<0.01 ;$ Fig. $3 \mathrm{~A})$ and those with GBM ( $\mathrm{P}=0.035$; Fig. $3 \mathrm{~B})$.

Multivariate analysis of 80 patients with GBM was performed for UBE2C expression and pathological predictors 
Table I. Association between UBE2C protein expression and clinical characteristics of patients with glioblastoma.

\begin{tabular}{lrcc}
\hline Characteristic & Negative & Positive & P-value \\
\hline Total, $\mathrm{n}$ & 17 & 63 & \\
Age, years & & & \\
$\quad<48$ & 10 & 23 & 0.16 \\
$>48$ & 7 & 40 & \\
Gender & & & \\
Male & 12 & 38 & 0.58 \\
Female & 5 & 25 & \\
Extent of resection & & & \\
Total & 4 & 28 & 0.17 \\
Subtotal & 13 & 35 & \\
IDH1 mutation status & & & \\
No mutation & 4 & 7 & 0.23 \\
Mutation & 13 & 56 & \\
TMZ chemotherapy & & & \\
No TMZ & 12 & 46 & 1.00 \\
TMZ & 5 & 17 & \\
\hline
\end{tabular}

Absent or weak staining was categorized as negative, while moderate or strong staining was categorized as positive. UBE2, ubiquitin-conjugating enzyme E2C; IDH1, isocitrate dehydrogenase-1; TMZ, temozolomide.

Table II. Association between UBE2C expression and clinical characteristics and the clinical outcome in 80 glioblastoma patients.

\begin{tabular}{|c|c|c|c|}
\hline Characteristic & HR & $95 \% \mathrm{CI}$ & P-value \\
\hline \multicolumn{4}{|l|}{ Gender } \\
\hline Male & 1.00 & & \\
\hline Female & 0.84 & $0.50-1.41$ & 0.51 \\
\hline \multicolumn{4}{|l|}{ Age, years } \\
\hline$<48$ & 1.00 & & \\
\hline$>48$ & 0.82 & $0.47-1.44$ & 0.49 \\
\hline \multicolumn{4}{|c|}{ Extent of resection } \\
\hline Subtotal & 1.00 & & \\
\hline Total & 0.44 & $0.25-0.77$ & $<0.01$ \\
\hline \multicolumn{4}{|c|}{ UBE2C expression } \\
\hline Low & 1.00 & & \\
\hline High & 2.40 & $1.23-4.69$ & 0.01 \\
\hline \multicolumn{4}{|c|}{ IDH1 mutation status } \\
\hline No mutation & 1.00 & & \\
\hline Mutation & 0.34 & $0.14-0.83$ & 0.02 \\
\hline \multicolumn{4}{|c|}{ TMZ chemotherapy } \\
\hline No TMZ & 1.00 & & \\
\hline TMZ & 0.37 & $0.20-0.70$ & $<0.01$ \\
\hline
\end{tabular}

Calculated using Cox's proportional hazard regression analysis. UBE2, ubiquitin-conjugating enzyme E2C; HR, hazard ratio; IDH1, isocitrate dehydrogenase-1; TMZ, temozolomide; CI, confidence interval. for survival time using Cox's proportional hazard regression analysis. The results demonstrated that the UBE2C expression, extent of resection, TMZ chemotherapy and IDH1 mutation status were independent prognostic factors of patient prognosis (Table II). Therefore, the expression level of UBEC1 may be a valuable prognostic marker for glioblastoma patients.

\section{Discussion}

Halting cell-cycle progression is crucial for the development of tumorigenesis and tumor progression (20). UBE2C is a human homolog of the cyclin-selective E2, which is involved in the destruction of mitotic cyclins (21). Previous studies have identified that, at the early G1 phase of the cell cycle, UBE2C undergoes anaphase-promoting, complex-dependent degradation $(22,23)$. This cell cycle-dependent expression of UBE2C may be a unique autoregulatory feedback loop for the regulation of antigen-presenting cells (24). Therefore, an impairment of UBE2C expression may inhibit the autoregulatory feedback loop and lead to the dysregulation of cell growth $(22,24,25)$. A previous study by van Ree et al (26) identified that UBE2C overexpression leads to early degradation of cyclin B, supernumerary centrioles, lagging chromosomes and aneuploidy. In addition, UBE2C transgenic mice were prone to carcinogen-induced lung tumors and a broad spectrum of spontaneous tumors (26). These results indicate that $\mathrm{UBE} 2 \mathrm{C}$ is a prominent protooncogene, which causes instability of the whole chromosome and leads to tumor formation.

The present study evaluated the expression levels of UBE2C in 220 human glioma tissues and 5 non-cancerous brain tissue specimens. The present study revealed that UBE2C was expressed at low levels in non-cancerous and low-grade glioma tissues, whereas high-grade gliomas expressed UBE2C at increased levels. This result was consistent with previous studies, which have revealed the involvement of UBE2C in the malignant transformation of tissues and tumor progression $(8,27,28)$. Okamoto et al $(24)$ observed that the expression levels of UBE2C were extremely low in numerous normal tissues, but prominent in the majority of primary tumors and cancerous cell lines. Additionally, an overexpression of UBE2C stimulated growth and colony-forming activity in NIH3T3 cells (24). In astrocytic tumors, UBE2C gene expression has been reported to increase with advancing pathological stages (17). Therefore, UBE2C may be vital in the aggressive progression of glioma.

However, the association between UBE2C expression and the clinical prognosis of glioma patients has not been defined. The present study demonstrated for the first time, to the best of our knowledge, that a high expression of UBE2C was associated with a poor outcome for patients with malignant gliomas. In the present study, 34 anaplastic gliomas and 89 GBM tissues were analyzed for UBE2C expression and survival time. The results demonstrated that anaplastic glioma and GBM patients with an increased UBE2C expression possessed a significantly decreased overall survival time. Multivariate analysis of 80 GBM patients indicated that UBE2C expression is an independent prognostic factor. These findings suggest that UBE2C may be a reliable indicator for prognosis in malignant glioma patients. 
In summary, the present study demonstrated that UBE2C is overexpressed in malignant glioma. This result is based on a large sample of frozen and FFPE tissue samples. The present study identified for the first time, to the best of our knowledge, that UBE2C is associated with an aggressive clinical outcome and decreased overall survival time. Multivariate regression analysis indicated the independence of UBE2C overexpression as a predictor for poor prognosis in malignant glioma patients. Additional investigation is required to confirm the findings prior to the clinical application of UBE2C.

\section{Acknowledgements}

The present study was supported by the Beijing Natural Science Foundation (grant no. 7154193).

\section{References}

1. Ricard D, Idbaih A, Ducray F, Lahutte M, Hoang-Xuan K and Delattre JY: Primary brain tumours in adults. Lancet 379: 1984-1996, 2012.

2. Fuller GN and Scheithauer BW: The 2007 revised World Health Organization (WHO) classification of tumours of the central nervous system: Newly codified entities. Brain Pathol 17: 304-307, 2007.

3. Talacchi A, Turazzi S, Locatelli F, Sala F, Beltramello A, Alessandrini F, Manganotti P, Lanteri P, Gambin R, Ganau M, et al: Surgical treatment of high-grade gliomas in motor areas. The impact of different supportive technologies: A 171-patient series. J Neurooncol 100: 417-426, 2010.

4. Nitta M, Muragaki Y, Maruyama T, Iseki H, Ikuta S, Konishi Y, Saito T, Tamura M, Chemov M, Watanabe A, et al: Updated therapeutic strategy for adult low-grade glioma stratified by resection and tumor subtype. Neurol Med Chir (Tokyo) 53: 447-454, 2013.

5. Martinho O, Granja S, Jaraquemada T, Caeiro C, Miranda-Gonçalves V, Honavar M, Costa P, Damasceno M, Rosner MR, Lopes JM and Reid RM: Downregulation of RKIP is associated with poor outcome and malignant progression in gliomas. PLoS One 7: e30769, 2012.

6. Hershko A and Ciechanover A: The ubiquitin system. Annu Rev Biochem 67: 425-479, 1998.

7. Joazeiro CA and Weissman AM: RING finger proteins: Mediators of ubiquitin ligase activity. Cell 102: 549-552, 2000.

8. Shen Z, Jiang X, Zeng C, Zheng S, Luo B, Zeng Y, Ding R, Jiang H, $\mathrm{He} \mathrm{Q}$, Guo J and Jie W: High expression of ubiquitin-conjugating enzyme $2 \mathrm{C}$ (UBE2C) correlates with nasopharyngeal carcinoma progression. BMC Cancer 13: 192, 2013.

9. Rape M and Kirschner MW: Autonomous regulation of the anaphase-promoting complex couples mitosis to S-phase entry. Nature 432: 588-595, 2004.

10. Rape M, Reddy SK and Kirschner MW: The processivity of multiubiquitination by the APC determines the order of substrate degradation. Cell 124: 89-103, 2006

11. Parris TZ, Danielsson A, Nemes S, Kovács A, Delle U, Fallenius G, Möllerström E, Karlsson P and Helou K: Clinical implications of gene dosage and gene expression patterns in diploid breast carcinoma. Clin Cancer Res 16: 3860-3874, 2010.

12. Lin J, Raoof DA, Wang Z, Lin MY, Thomas DG, Greenson JK, Giordano TJ, Orringer MB, Chang AC, Beer DG and Lin L: Expression and effect of inhibition of the ubiquitin-conjugating enzyme E2C on esophageal adenocarcinoma. Neoplasia 8: 1062-1071, 2006.
13. Bavi P,Uddin S, Ahmed M, Jehan Z, Bu R, Abubaker J, Sultana M, Al-Sanea N, Abduljabbar A, Ashari LH, et al: Bortezomib stabilizes mitotic cyclins and prevents cell cycle progression via inhibition of UBE2C in colorectal carcinoma. Am J Pathol 178: 2109-2120, 2011 .

14. Wagner KW, Sapinoso LM, El-Rifai W, Frierson HF, Butz N, Mestan J, Hofmann F, Deveraux QL and Hampton GM: Overexpression, genomic amplification and therapeutic potential of inhibiting the $\mathrm{UbcH} 10$ ubiquitin conjugase in human carcinomas of diverse anatomic origin. Oncogene 23: 6621-6629, 2004.

15. Ieta K, Ojima E, Tanaka F, Nakamura Y, Haraguchi N, Mimori K, Inoue $\mathrm{H}$, Kuwano $\mathrm{H}$ and Mori M: Identification of overexpressed genes in hepatocellular carcinoma, with special reference to ubiquitin-conjugating enzyme E2C gene expression. Int J Cancer 121: 33-38, 2007.

16. Berlingieri MT, Pallante P, Guida M, Nappi C, Masciullo V, Scambia G, Ferraro A, Leone V, Sboner A, Barbareschi M, et al: $\mathrm{UbcH} 10$ expression may be a useful tool in the prognosis of ovarian carcinomas. Oncogene 26: 2136-2140, 2007.

17. Donato G, Iofrida G, Lavano A, Volpentesta G, Signorelli F, Pallante PL, Berlingieri MT, Pierantoni MG, Palmieri D, Conforti F, et al: Analysis of UbcH10 expression represents a useful tool for the diagnosis and therapy of astrocytic tumors. Clin Neuropathol 27: 219-223, 2008.

18. Jiang L, Huang CG, Lu YC, Luo C, Hu GH, Liu HM, Chen JX and Han HX: Expression of ubiquitin-conjugating enzyme E2C/UbcH10 in astrocytic tumors. Brain Res 1201: 161-166, 2008.

19. Zhang W, Qiu XG, Chen BS, Li SW, Cui Y, Ren H and Jiang T: Antiangiogenic therapy with bevacizumab in recurrent malignant gliomas: Analysis of the response and core pathway aberrations. Chin Med J (Engl) 122: 1250-1254, 2009.

20. Jiang L, Wang T, Bao Y, Qian J, Wu XJ, Hu GH and Lu YC: A study of UbcH10 expression and its association with recurrence of meningiomas. J Surg Oncol 106: 327-331, 2012.

21. Townsley FM, Aristarkhov A, Beck S, Hershko A and Ruderman JV: Dominant-negative cyclin-selective ubiquitin carrier protein E2-C/UbcH10 blocks cells in metaphase. Proc Natl Acad Sci USA 94: 2362-2367, 1997.

22. Yamanaka A, Hatakeyama S, Kominami K, Kitagawa M, Matsumoto M and Nakayama K: Cell cycle-dependent expression of mammalian E2-C regulated by the anaphase-promoting complex/cyclosome. Mol Biol Cell 11: 2821-2831, 2000.

23. Fujita T, Ikeda H, Taira N, Hatoh S, Naito M, Doihara H: Overexpression of $\mathrm{UbcH} 10$ alternates the cell cycle profile and accelerate the tumor proliferation in colon cancer. BMC Cancer 9: 87, 2009.

24. Okamoto Y, Ozaki T, Miyazaki K, Aoyama M, Miyazaki M and Nakagawara A: UbcH10 is the cancer-related E2 ubiquitin-conjugating enzyme. Cancer Res 63: 4167-4173, 2003.

25. Troncone G, Guerriero E, Pallante P, Berlingieri MT, Ferraro A, Del Vecchio L, Gorrese M, Mariotti E, Iaccarino A, Palmieri $\mathrm{EA}$, et al: $\mathrm{UbcH} 10$ expression in human lymphomas. Histopathology 54: 731-740, 2009.

26. van Ree JH, Jeganathan KB, Malureanu L and van Deursen JM: Overexpression of the $\mathrm{E} 2$ ubiquitin-conjugating enzyme UbcH10 causes chromosome missegregation and tumor formation. J Cell Biol 188: 83-100, 2010.

27. Matsumoto A, Ishibashi Y, Urashima M, Omura N, Nakada K, Nishikawa K, Shida A, Takada K, Kashiwagi H and Yanaga K: High UBCH10 protein expression as a marker of poor prognosis in esophageal squamous cell carcinoma. Anticancer Res 34: 955-961, 2014

28. Li SZ, Song Y, Zhang HH, Jin BX, Liu Y, Liu WB, Zhang XD and Du RL: UbcH10 overexpression increases carcinogenesis and blocks ALLN susceptibility in colorectal cancer. Sci Rep 4: 6910, 2014. 